\title{
Strategic manipulations of multi-valued solutions in economies with indivisibilities *
}

\author{
Olivier Bochet ${ }^{\dagger} \quad$ Toyotaka Sakai $^{\ddagger}$
}

June 7, 2004

\begin{abstract}
We study manipulability of multi-valued solutions in fair allocation of homogeneous indivisible objects with monetary transfers. We show that the no-envy solution satisfies some extension of strategy-proofness to multi-valued solutions. Since no-envy implies Pareto efficiency in this literature, this result implies that we can escape many impossibility results on strategy-proofness by allowing a solution to be multi-valued and extending strategy-proofness in an appropriate way. Nevertheless, we show that no-envy is not immune to all forms of manipulations. In that sense, we are able to identify what kind of manipulations occur. Next, we derive impossibility results on the egalitarian-equivalence and efficient solution. The intuition that manipulability comes from singlevaluedness is not confirmed by this solution. Finally, we show that there is no subsolution of the Pareto and identical preferences lower bound satisfying the notion of extended strategy-proofness used by TadenumaThomson (1995).
\end{abstract}

Keywords: Indivisible goods, Extended strategy-proofness, Noenvy, Egalitarian-equivalence, Identical preferences lower bound, Fair allocation.

${ }^{*}$ The first version was written while T. Sakai was visiting the University of Namur. He gratefully acknowledges the hospitality of the school.

${ }^{\dagger}$ University of Namur and CORE, 8 rempart de la vierge, 5000 Namur, Belgium; olivier.bochet@fundp.ac.be.

$\ddagger$ Department of Economics, University of Rochester, Rochester, NY 14627, USA; toyo@troi.cc.rochester.edu; http://troi.cc.rochester.edu/ toyo. 


\section{Introduction}

We consider the problem of fairly allocating homogeneous indivisible goods when monetary compensations are possible and preferences are quasi-linear. A solution is a correspondence which associate each preference profile with a non-empty set of feasible allocations. Our purpose is to seek for solutions that are immune to some form of strategic manipulations.

A central property on non-manipulability is strategy-proofness, which states that no one can gain by misrepresenting his preference. Strategy-proofness is a desirable property because it requires very little on behalf of the agents. In the direct revelation mechanism, reporting truthfully his own preferences is a (weakly) dominant strategy. The only thing that each agent needs to know -apart from the obvious knowledge of the game form and of the possible outcomes associated with it- is that she is rational. No assumptions on the behavior of other agents is necessary. However, it is known that in this context, no reasonable solution is strategy-proof (Schummer, 1999; Svensson, 2000; Ohseto, 2000, 2003). On the other hand, strategy-proofness is welldefined only for (essentially ${ }^{1}$ ) single-valued solutions, and we have few wellbehaved single-valued solutions in this literature. Therefore, the assumption of single-valuedness itself is restrictive. We consider that many impossibility results on strategy-proofness may come from the underlying assumption of single-valuedness rather than the property of strategy-proofness. The interest in manipulability of multi-valued rules is not new. The first paper is Gibbard (?). He considers correspondences but reduces them to functions by taking lotteries over alternatives selected. He obtains a random dictatorship theorem. Related to Gibbard's result, Barbera (1977), Kelly (1977) and Barbera-DuttaSen (2001) also examine the manipulability of multi-valued rules. To examine our question, we extend the definition of strategy-proofness to multi-valued solutions in the following manner: Given a true preference profile, any allocation that an agent can add to the solution by misrepresenting his preference is worse than all allocations chosen under the true preference profile. Let us call this property $^{2}$ extended strategy-proofness II. We also review some extensions that have been proposed in the literature (Ching-Zhou, 1991; Tadenuma-Thomson,

\footnotetext{
${ }^{1} \mathrm{~A}$ solution is essentially single-valued if every alternatives are welfare equivalent. That is, each agent is indifferent between all the alternatives selected.

${ }^{2}$ In the definitions, we list the various extended strategy-proofness conditions starting with the weakest. Strategy resistance (Jackson, 1992) is the weakest and ours comes next, hence the name of extended strategy-proofness II. We list Tadenuma-Thomson's condition as extended strategy-proofness III though it cannot be compared to ours. However, both extended strategy-proofness II and extended strategy-proofness III imply strategy-resistance. Ching-Zhou comes last and is strongest than extended strategy-proofness II and extended strategy-proofness III as shown in lemma 1.
} 
1996 and Jackson (1992)) and compare them to our definition. All these extensions, including ours, coincide with strategy-proofness under the assumption of single-valuedness.

Two solutions have played a central role in this literature. The no-envy solution associates each preference profile with a set of feasible allocations at which no agent prefers the consumption bundle of any other to his own. In addition to its direct normative significance, the no-envy solution is quite desirable in that any envy-free allocation is Pareto efficient (Svensson, 1983) and meets an "identical preferences lower bound" (Bevia, 1996). The egalitarianequivalent efficient (Pazner-Schmeidler, 1978) solution associates each preference profile with a set of feasible and efficient allocations which has the property that, for each allocation selected, agents are indifferent between the bundle they are receiving and a common reference bundle.

First, we show that the no-envy solution satisfies extended strategy-proofness II. As a corollary, it implies that strategy-resistance (Jackson, 1992) is also satisfied. This result strengthens the appeal of the no-envy solution from the viewpoint of non-manipulability. Our result contrasts with many impossibility results on strategy-proofness for single-valued solutions. In particular, Ohseto (2000) shows that, when there is only one indivisible good, even on finitely restricted preference domains, there is no single-valued solution satisfying strategy-proofness, Pareto efficiency, and equal compensation (every two agents who do not receive the indivisible good should receive the same amount of money). Since no-envy implies both Pareto efficiency and equal compensation, our result suggests that we can escape Ohseto's impossibility result if we allow multi-valuedness. Next, we show that there is no subsolution of the Pareto and identical preferences lower bound solution ${ }^{3}$ that satisfies Tadenuma-Thomson's definition of non- manipulability.

We also examine another form of manipulations in which an agent reduces undesirable part of allocations chosen under a true preference profile by misrepresenting his preference. We call this reduction-manipulation. We show that $^{4}$, for each preference profile, there exists an agent who can reductionmanipulate the no-envy solution. This result also implies that Ching-Zhou's notion of non-manipulability cannot be satisfied.

Hence, by enlarging the solution from single-valued to multi-valued, we can escape negative results. It entails believing that our extended strategyproofness condition is a sensible one. Single-valuedness may therefore be too

\footnotetext{
${ }^{3}$ Note that the no-envy solution is contained in the Pareto and identical preferneces lower bound solution.

${ }^{4}$ Under the restriction that among the agents who receive a real object, the one with the lowest valuation has a valuation strictly greater than the agent who has the highest valuation among those who receive a null-object.
} 
strong a requirement.

Next, we examine the manipulability of the egalitarian-equivalent solution. We show that no subsolution of the egalitarian-equivalent efficient solution satisfy strategy-resistance. This result is interesting in that it shows a robustness of the manipulability of this solution. It is often viewed that rules are manipulable essentially because they are single-valued. By extending strategy-proofness to correspondences, the previous result on the no-envy solution sustains that conclusion. On the other hand, it is not confirmed with the egalitarian-equivalent efficient solution. The manipulability problem inherent to egalitarian-equivalent efficient allocations is therefore not related to single-valuedness.

Finally, we also show that in the two agent case, there is no subsolution of the Pareto solution satisfying Ching-Zhou's notion of non-manipulability, equal treatment of equals and neutrality.

The paper is organized as follows: Section 2 introduces definitions. Section 3 provides the extended strategy proofness conditions we use. Section 4 contains characterization of the set of no-envy and the set of egalitarianequivalent allocations. Our main results on the manipulability of fair solutions is in section 5. Finally, section 6 concludes the discussion.

\section{Definitions}

Let $N \equiv\{1,2, \ldots, n\}$ be a finite set of agents. There are $l(1 \leq l \leq n-1)$ units of homogeneous indivisible good, $\alpha$. For convenience, we consider that any agent who receives no indivisible good receives a "null" good, $\nu$. We allow transfers of money among the agents. An allocation is a pair

$$
z \equiv\left(z_{i}\right)_{i \in N} \equiv\left(\sigma(i), m_{i}\right)_{i \in N} \equiv(\sigma, m),
$$

where $\sigma: N \rightarrow\{\alpha, \nu\}$ is a function such that $\left|\sigma^{-1}(\alpha)\right|=l$ and $m \in \mathbb{R}^{N}$ is such that $\sum_{i \in N} m_{i}=0$. Here $\sigma(i)$ is the indivisible object that $i$ receives and $m_{i} \geq 0$ (resp. $m_{i}<0$ ) is the amount of money he is paid (resp. pays). Let $Z$ be the set of allocations.

Each agent $i \in N$ has a quasi-linear preference $R_{i}$ over the consumption space $\{\alpha, \nu\} \times \mathbb{R}$, which is characterized by a single number $v_{i} \in \mathbb{R}$ such that for each $m_{i} \in \mathbb{R},\left(\alpha, m_{i}\right) I_{i}\left(\nu, v_{i}+m_{i}\right)$. Let $\mathcal{R}$ be the set of quasi-linear preferences. A preference profile is an $n$-tuple of preferences $R \equiv\left(R_{1}, R_{2}, \ldots, R_{n}\right) \in \mathcal{R}^{N}$. Given $R \in \mathcal{R}^{N}$, an agent $i$ is an upper threshold at $R$ if his evaluation $v_{i}$ is $n$-th highest, i.e., $\left|\left\{v_{j} \in\left\{v_{1}, v_{2}, \ldots, v_{n}\right\}: v_{j} \geq v_{i}\right\}\right|=\ell$. Similarly, $i$ is a lower threshold at $R$ if his evaluation is $n-\ell$ lowest, i.e., $\mid\left\{v_{j} \in\left\{v_{1}, v_{2}, \ldots, v_{n}\right\}\right.$ : $\left.v_{j} \leq v_{i}\right\} \mid=n-\ell$. Let $\bar{v}, \underline{v} \in \mathbb{R}$ be the evaluations of the upper and lower thresholds at $R$, respectively. 
A solution is a correspondence from $\mathcal{R}^{N}$ to $Z$ which associates a profile $R \in \mathcal{R}^{N}$ a non-empty set of allocations, $\psi(R) \subseteq Z$. The following is a familiar efficiency solution:

The Pareto solution, $P$ : An allocation $z \in Z$ is Pareto efficient for $R \in \mathcal{R}^{N}$, if there exists no $z^{\prime} \in Z$ such that for each $i \in N, z_{i}^{\prime} R_{i} z_{i}$ and for some $j \in N$, $z_{j}^{\prime} P_{j} z_{j}$. Given $R \in \mathcal{R}^{N}$, let $P(R)$ be the set of Pareto efficient allocations for $R$.

By quasi-linearity of preferences, an allocation $(\sigma, m)$ is Pareto efficient for $R$ if and only if for each $i, j \in N$ such that $\sigma(i)=\alpha$ and $\sigma(j)=\nu$, we have $v_{i} \geq v_{j}$.

The next solution associates each preference profile to the set of allocations at which everyone weakly prefers his own bundle to anyone else's bundle:

The no-envy solution, $\boldsymbol{F}:$ An allocation $z \in Z$ is envy-free for $R \in \mathcal{R}^{N}$, if for each $i, j \in N, z_{i} R_{i} z_{j}$. Given $R \in \mathcal{R}^{N}$, let $F(R)$ be the set of envy-free allocations for $R$.

Under our assumptions on preferences, the no-envy solution is well-defined (Alkan, Demange, and Gale, 1991, Theorem 2). ${ }^{5}$ In this context, the no-envy solution is a subsolution of the Pareto solution (Svensson, 1983, Theorem 2). This relation much strengthens the appeal of the no-envy solution in that fairness as envy-free is completely consistent with Pareto efficiency.

The notion of egalitarian equivalence (Pazner and Schmeidler, 1978) states that each agent should receive a consumption bundle that is indifferent to a common "reference" consumption bundle. Our purpose is to examine the robustness of egalitarian-equivalent solutions to strategic manipulations. Given $R \in \mathcal{R}$, an allocation $z$ is $\alpha$-egalitarian equivalent for $R$ if there exists $m_{\alpha}^{*} \in \mathbb{R}$ such that for each $i \in N, z_{i} I_{i}\left(\alpha, m_{\alpha}^{*}\right)$. Let $E^{\alpha}(R)$ be the set of $\alpha$-egalitarian equivalent allocations for $R$. Similarly, $z$ is $\nu$-egalitarian equivalent for $R$ if there exists $m_{\nu}^{*} \in \mathbb{R}$ such that for each $i \in N, z_{i} I_{i}\left(\alpha, m_{\nu}^{*}\right)$. Let $E^{\nu}(R)$ be the set of $\nu$-egalitarian equivalent allocations for $R$.

Egalitarian-equivalent solution, $\boldsymbol{E}$ : For each $R \in \mathcal{R}$, let $E(R) \equiv E^{\alpha}(R) \cup$ $E^{\nu}(R)$ be the set of egalitarian equivalent allocations.

The notion of identical preferences lower bound states that everyone should benefit from the diversity of preferences (Moulin, 1990). Given $R_{i} \in \mathcal{R}$, let $r\left(R_{i}\right) \in Z$ be a "reference" allocation for $R_{i}$ : it is the Pareto efficient allocation for the economy with the identical preference $R_{i}$ such that for each $j \in N$,

\footnotetext{
${ }^{5}$ The existence can be derived as a corollary to one of our lemmas that characterizes envy-free allocations (see, Lemma 2).
} 
$r_{i}\left(R_{i}\right) I_{i} r_{j}\left(R_{i}\right)$. Note that such an $r\left(R_{i}\right)$ is essentially unique in that if $r\left(R_{i}\right)$ and $r^{\prime}\left(R_{i}\right)$ are two reference allocations, $r_{i}\left(R_{i}\right) I_{i} r_{i}^{\prime}\left(R_{i}\right)$. Slightly abusing language, we deal with $r\left(R_{i}\right)$ as if it were a consumption bundle.

Identical preferences lower bound solution, $\underline{B}_{i p}$ : For each $R \in \mathcal{R}$, let $\underline{B}_{i p}(R) \equiv\left\{z \in Z:\right.$ For each $\left.i \in N, z_{i} R_{i} r\left(R_{i}\right)\right\}$.

It is known that if $n=2$, then $F=\underline{B}_{i p}$, and if $n>2$, then $F \subsetneq \underline{B}_{i p}$ (Bevia, 1996, Propositions 1 and 2).

\section{$3 \quad$ Extensions of strategy-proofness}

We begin by introducing the central property on manipulability of singlevalued solutions: no one can gain by misrepresenting his preference.

Strategy-proofness (for single-valued solutions): For each $R \in \mathcal{R}^{N}$, each $i \in N$, each $R_{i}^{\prime} \in \mathcal{R}$, and $z \in \psi(R), z R_{i} \psi\left(R_{i}^{\prime}, R_{-i}\right)$.

We present four extensions of strategy-proofness to multi-valued solutions. They coincide with strategy-proofness if solutions are single-valued. The first property states that any allocation obtained by a misrepresentation is not better than an originally chosen allocation (Jackson, 1992) ${ }^{6}$ :

Extended strategy-proofness I: For each $R \in \mathcal{R}^{N}$, each $i \in N$, each $R_{i}^{\prime} \in$ $\mathcal{R}$, and each $z^{\prime} \in \psi\left(R_{i}^{\prime}, R_{-i}\right)$, there exists $z \in \psi\left(R_{i}, R_{-i}\right)$ such that $z_{i} R_{i} z_{i}^{\prime}$.

Given a preference $R_{i} \in \mathcal{R}$ and a set of allocations $Z^{\prime} \subseteq Z$, we write $\min \left(R_{i}, Z^{\prime}\right) R_{i} z_{i}$ if for each $x \in Z^{\prime}, x_{i} R_{i} z_{i}$. Consider an allocation obtained by a misrepresentation that is not originally chosen. The next property states that such an allocation is worse than all of the originally chosen allocations:

Extended strategy-proofness II: For each $R \in R^{N}$, each $i \in N$, each $R_{i}^{\prime} \in R$, and each $z^{\prime} \in \psi\left(R_{i}^{\prime}, R_{-i}\right)$, if $z^{\prime} \notin \psi(R)$, then $\min \left(R_{i}, \psi(R)\right) R_{i} z_{i}^{\prime}$.

The next property prohibits the following situation: at any chosen allocation, there exists an agent such that if he misrepresents his preference, then he can gain at all allocations chosen under the revised preference profile (Hurwicz, 1972; Tadenuma and Thomson, 1995):

Extended strategy-proofness III: For each $R \in \mathcal{R}^{N}$, there exists $z \in \psi(R)$ such that for each $i \in N$ and each $R_{i}^{\prime} \in \mathcal{R}$, there exists $z^{\prime} \in \psi\left(R_{i}^{\prime}, R_{-i}\right)$ for

\footnotetext{
${ }^{6}$ Jackson calls this property "strategy-resistance". He shows that this condition is necessary for a solution to be fully implementable in undominated strategies using bounded mechanisms.
} 
which $z_{i} R_{i} z_{i}^{\prime}$.

A reformulation of extended strategy-proofness in terms of manipulation may be beneficial: there exists no $R \in \mathcal{R}^{N}$ such that for each $z \in \psi(R)$, there exist $i \in N$ and $R_{i}^{\prime} \in \mathcal{R}$ for which for each $z^{\prime} \in \psi\left(R_{i}^{\prime}, R_{-i}\right), z_{i}^{\prime} P_{i} z_{i}$.

The following property consists of two parts (Ching and Zhou, 2002): the first part states that when an agent removes a chosen allocation by a misrepresentation, then such an allocation is worse than all of chosen allocations under the misreported profile; the second part is extended strategy-proofness II.

Extended strategy-proofness IV: For each $R \in \mathcal{R}^{N}$, each $i \in N$, and each $R_{i}^{\prime} \in \mathcal{R}$,

1) for each $z \in \psi(R) \backslash \psi\left(R_{i}^{\prime}, R_{-i}\right)$ and each $z^{\prime} \in \psi\left(R_{i}^{\prime}, R_{-i}\right), z_{i} R_{i} z_{i}^{\prime}$,

2) for each $z^{\prime} \in \psi\left(R_{i}^{\prime}, R_{-i}\right) \backslash \psi(R)$ and each $z \in \psi(R), z_{i} R_{i} z_{i}^{\prime}$.

We introduce a manipulability notion that is specific to multi-valued solutions. An agent $i$ can reduction-manipulate a solution $\psi$ at $R \in \mathcal{R}^{N}$ if there exists $R_{i}^{\prime} \in \mathcal{R}$ such that $\psi\left(R_{i}^{\prime}, R_{-i}\right) \subsetneq \psi(R)$ and for each $z \in \psi(R) \backslash \psi\left(R_{i}^{\prime}, R_{-i}\right)$, $\min \left(R_{i}, \psi\left(R_{i}^{\prime}, R_{-i}\right)\right) P_{i} z_{i}$.

Reduction-non-manipulability: There exist no $i \in N$ and $R \in \mathcal{R}^{N}$ such that $i$ can reduction manipulate $\psi$ at $R$.

The next lemma summarized logical relations between the axioms defined above:

Lemma 1. (i) Extended strategy-proofness $I V$ implies both $I I$ and $I I I$, and each of $I I$ and $I I I$ implies $I$;

(ii) Extended strategy-proofness III implies reduction-non-manipulability, while Extended strategy-proofness II does not.

Proof. (i) is easy, so omitted. For (ii), it is also easy to see that extended strategy-proofness III implies reduction-non-manipulability. The no-envy solution satisfies extended strategy-proofness II but is reduction-manipulable (see, Theorems 2 and 3 in Section 5).

\section{Preliminary results}

We provide characterizations of the sets of envy-free allocations and egalitarian equivalent allocations. These results are useful to understand how the set of chosen allocations changes according to the misrepresentation of a preference.

Lemma 2. For each $R \in \mathcal{R}^{N}$, an allocation $z=(\sigma, m) \in Z$ is envy-free if and only if there exists a partition of $N,\left\{N^{\prime}, N^{\prime \prime}\right\}$, such that 
(i) for each $i, j \in N^{\prime}, \sigma(i)=\alpha$ and $m_{i}=m_{j}$,

(ii) for each $i, j \in N^{\prime \prime}, \sigma(i)=\nu$ and $m_{i}=m_{j}$,

(iii) when $k=\arg \min _{i \in N^{\prime}} v_{i}$ and $h=\arg \max _{i \in N^{\prime \prime}} v_{i}$, we have $v_{k} \geq v_{h}$,

$$
m_{k} \in\left[-\frac{n-\ell}{n} v_{k},-\frac{n-\ell}{n} v_{h}\right] \text { and } m_{h} \in\left[\frac{\ell}{n} v_{h}, \frac{\ell}{n} v_{k}\right] .7
$$

Proof. Let $z \equiv(\sigma, m) \in F(R)$. Let $N^{\prime} \equiv\{i \in N: \sigma(i)=\alpha\}$ and $N^{\prime \prime} \equiv\{i \in$ $N: \sigma(i)=\nu\}$. Since $z \in F(R)$, both (i) and (ii) hold. Let us verify (iii). Since $z \in P(R)$, when $k=\arg \min _{i \in N^{\prime}} v_{i}$ and $h=\arg \max _{i \in N^{\prime \prime}} v_{i}$, we have $v_{k} \geq v_{h}$. Note that $\left(\alpha,-\frac{n-\ell}{n} v_{k}\right) I_{k}\left(\nu, \frac{\ell}{n} v_{k}\right)$ and $\left(\alpha,-\frac{n-\ell}{n} v_{h}\right) I_{h}\left(\nu, \frac{\ell}{n} v_{h}\right)$. If $-\frac{n-\ell}{n} v_{k}>$ $m_{k}$, then agent $k$ envies agent $h$. If $m_{k}>-\frac{n-\ell}{n} v_{h}$, then agent $h$ envies agent $k$. Therefore, $m_{k} \in\left[-\frac{n-\ell}{n} v_{k},-\frac{n-\ell}{n} v_{h}\right]$, and by feasibility, $m_{h} \in\left[\frac{\ell}{n} v_{h}, \frac{\ell}{n} v_{k}\right]$.

Conversely, let $z \in Z$ satisfy (i)-(iii) associated with a partition $\left\{N^{\prime}, N^{\prime \prime}\right\}$. Let $i, j \in N$. If either $i, j \in N^{\prime}$ or $i, j \in N^{\prime \prime}$, then by (i) and (ii), $z_{i} I_{i} z_{j}$. Hence, suppose that $i \in N^{\prime}$ and $j \in N^{\prime \prime}$. When $k=\arg \min _{i \in N^{\prime}} v_{i}$ and $h=$ $\arg \max _{i \in N^{\prime \prime}} v_{i}$, by $v_{i} \geq v_{k}$ and (iii), $\left(\alpha, m_{i}\right) R_{i}\left(\alpha,-\frac{n-\ell}{n} v_{k}\right) R_{i}\left(\nu,-\frac{\ell}{n} v_{k}\right) R_{i}$ $\left(\nu, m_{j}\right)$. Similarly, by $v_{h} \geq v_{j}$ and (iii), $\left(\nu, m_{j}\right) R_{j}\left(\nu, \frac{\ell}{n} v_{h}\right) R_{i}\left(\alpha,-\frac{n-\ell}{n} v_{h}\right) R_{i}$ $\left(\alpha, m_{i}\right)$. Therefore, $z \in F(R)$.

When $z, z^{\prime} \in F(R)$, if agents receiving $\alpha$ prefer $z_{i}$ to $z_{i}^{\prime}$, agents receiving $\nu$ prefer $z_{i}^{\prime}$ to $z_{i}$, and vice versa. Hence, there are the " $\alpha$-optimal" and " $\nu$ optimal" envy-free allocations for $R$. In language of the above lemma, these allocations are characterized by $m_{k}=-\frac{n-\ell}{n} v_{h}$ and $m_{h}=\frac{\ell}{n} v_{k}$, respectively. Lemma 2 states that the $\alpha$-optimal (resp. $\nu$-optimal) envy-free allocation is determined by the preference of the agent whose evaluation is highest among those who receive the null object (resp. real object). This fact suggests that no agent who receives $\alpha$ can manipulate the no-envy solution in such a way that the $\alpha$-optimal envy-free allocation is improved. Also, it suggests that enlarging the set of envy-free allocations in a favored way is impossible. These intuitions are confirmed by the following possibility theorem:

Next we discuss the egalitarian equivalent solution and its subsolutions. The following is a characterization of the set of egalitarian-equivalent allocations:

Lemma 3. For each $R \in \mathcal{R}^{N}, z \in Z$ is $\alpha$-egalitarian equivalent for $R$ if and only if for each $i \in N^{\alpha}(z), m_{i}=-\frac{\sum_{j \in N^{\nu}(z)} v_{j}}{n}$, and for each $i \in N^{\nu}(z)$, $m_{i}=v_{i}-\frac{\sum_{j \in N^{\nu}(z)} v_{j}}{n}$.

\footnotetext{
${ }^{7}$ Tadenuma and Thomson (1995, Lemma 1) provides the same characterization when there is only one real object.
} 
Proof. It is easy to see that if $z \in Z$ is such that for each $i \in N^{\alpha}(z), m_{i}=$ $-\frac{\sum_{j \in N^{\nu}(z)} v_{j}}{n}$, and for each $i \in N^{\nu}(z)$, then it is $\alpha$-egalitarian equivalent for $R$.

Conversely, let $z \equiv(\sigma, m) \in Z$ be $\alpha$-egalitarian equivalent for $R$. Without loss of generality, assume that $N^{\nu}(z)=\{1,2, \ldots, n-l\}$ and $v_{1} \leq v_{2} \leq \cdots \leq$ $v_{n-l}$. Since $z$ is $\alpha$-egalitarian equivalent, all agents in $N^{\alpha}(z)$ receive the same amount of money, $x \in \mathbb{R}$. For each $i \in N^{\nu}(z)$, since $(\alpha, x) I_{i}\left(\nu, m_{i}\right), m_{i}-v_{i}=$ $x$. Note that for each $i, j \in N^{\nu}(z)$ with $v_{i} \leq v_{j}, m_{j}$ is greater than $m_{i}$ exactly by $v_{j}-v_{i}$. Therefore,

$$
\begin{array}{r}
\quad \sum_{i=1}^{n-l} m_{i}=m_{1}+\left(m_{1}+\left(v_{2}-v_{1}\right)\right)+\left(m_{1}+\left(v_{3}-v_{1}\right)\right)+\cdots+\left(m_{1}+\left(v_{n-l}-v_{1}\right)\right) \\
=(n-l) m_{1}+\sum_{i=2}^{n-l} v_{i}-(n-l-1) v_{1}=(n-l)\left(m_{1}-v_{1}\right)+\sum_{i=1}^{n-l} v_{i}=(n-l) x+\sum_{i=1}^{n-l} v_{i} .
\end{array}
$$

By budget balancedness, $(n-l) x+\sum_{i=1}^{n-l} v_{i}+l x=0$. Hence, $x=-\frac{\sum_{i=1}^{n-l} v_{i}}{n}$.

This lemma implies that any $\alpha$-egalitarian equivalent allocation is characterized by valuations of agents who receive $\nu$. Thus, whenever the set $N^{\nu}(z)$ is unchanged, any agent $i \in N^{\nu}(z)$ can increase his money by reporting $v_{i}^{\prime}>v_{i}$. This fact suggests the difficulty of egalitarian-equivalent solutions on the satisfaction of our strategy-proofness axioms. Since $\alpha$-egalitarian equivalence and $\nu$-egalitarian equivalence are symmetric, the characterization of $\nu$-egalitarian equivalent allocations is also obtained.

Lemma 4. For each $R \in \mathcal{R}^{N}, z \in Z$ is $\nu$-egalitarian equivalent for $R$ if and only if for each $i \in N^{\alpha}(z), m_{i}=v_{i}-\frac{\sum_{j \in N^{\alpha}(z)} v_{j}}{n}$, and for each $i \in N^{\nu}(z)$, $m_{i}=-\frac{\sum_{j \in N^{\alpha}(z)} v_{j}}{n}$.

The following is a characterization of reference bundles for the identical preferences lower bound:

Lemma 5. For each $R_{i} \in \mathcal{R}, r\left(R_{i}\right)=\left(\alpha,-\frac{n-\ell}{n} v_{i}\right)$ (or, alternately, $\left(\nu, \frac{\ell}{n} v_{i}\right)$ ).

Proof. Since the set of allocations that meet equal treatment of equals for $\left(R_{i}, R_{i}, \ldots, R_{i}\right)$ coincides with the set of envy-free allocations $F\left(R_{i}, R_{i}, \ldots, R_{i}\right)$, this immediately follows from Lemma 2.

\section{$5 \quad$ Manipulability of fair solutions}

We first show that various subsolutions of the egalitarian equivalent solution are not robust to strategic manipulations. They cannot even satisfy extended strategy-proofness $I$. 
Theorem 1. The following solutions do not satisfy extended strategy-proofness $I$ : the egalitarian-equivalent solution, the $\alpha$-egalitarian equivalent solution, the $\nu$-egalitarian equivalent solution, and all subsolutions of the Pareto and egalitarian equivalent solution.

Proof. It is obvious by Lemmas 3 and 5 that none of $E, E^{\alpha}$, and $E^{\nu}$ satisfies extended strategy-proofness $I$.

Suppose, by contradiction, that there exists $\psi \subseteq P E$ satisfying strategyresistance. Let $R \in \mathcal{R}^{N}$ be such that $v \equiv(0,0, \ldots, 0)$. Let $z \in \psi(R)$. Without loss of generality, we can assume that

$$
z=(\underbrace{(\alpha, 0), \ldots,(\alpha, 0)}_{l}, \underbrace{(\nu, 0), \ldots,(\nu, 0)}_{n-l}) .
$$

Note that $\max \left(R_{1}, \psi(R)\right)=(\alpha, 0)$.

Let $R^{\prime} \in \mathcal{R}^{N}$ be such that $v^{\prime} \equiv(1,0, \ldots, 0)$. We claim that every $z^{\prime} \in \psi\left(R^{\prime}\right)$ is $\alpha$-egalitarian equivalent. Indeed, if there exists $z^{\prime} \in \psi\left(R^{\prime}\right)$ that is $\nu$ egalitarian equivalent, then $z_{1}^{\prime}=\left(\alpha, 1-\frac{1}{n}\right)$. However, then agent 1 can manipulate $\psi$ at $v$ via $v^{\prime}$. Therefore, $\psi\left(R^{\prime}\right) \subseteq E^{\alpha}\left(R^{\prime}\right)$. Without loss of generality, assume that agent $n$ is such that for some $z^{\prime} \in \psi\left(R^{\prime}\right), z_{n}^{\prime}=(\nu, 0)$.

Let $R^{\prime \prime} \in \mathcal{R}^{N}$ be such that $v^{\prime} \equiv(1,0, \ldots,-1)$. Let $z^{\prime \prime} \in \psi\left(R^{\prime \prime}\right)$. If $z^{\prime \prime}$ is $\alpha$-egalitarian equivalent for $R^{\prime \prime}$, then $z_{n}^{\prime \prime}=\left(\nu,-1+\frac{1}{n}\right)$. If $z^{\prime \prime}$ is $\nu$-egalitarian equivalent for $R^{\prime \prime}$, then $z_{n}^{\prime \prime}=\left(\nu, \frac{1}{n}\right)$. Therefore, $z_{n}^{\prime} P_{n}^{\prime \prime} \max \left(R_{n}^{\prime}, \psi\left(R^{\prime \prime}\right)\right)$. This implies that agent $n$ can manipulate $\psi$ at $v^{\prime \prime}$ via $v^{\prime}$. This is a contradiction.

In view of Theorem 1, a natural question is whether the no-envy solution satisfies extended strategy-proofness I. Our answer is positive. The no-envy solution in fact satisfies more stronger version:

Theorem 2. The no-envy solution satisfies extended strategy-proofness II.

Proof. Let $z \in F(R)$. Without loss of generality, assume that $v_{1} \leq v_{2} \leq \cdots \leq$ $v_{n}$,

$$
N^{\prime} \equiv\left\{v_{k}, v_{k+1}, \ldots, v_{n}\right\}=\{i \in N: \sigma(i)=\alpha\}
$$

and

$$
N^{\prime \prime} \equiv\left\{v_{1}, v_{2}, \ldots, v_{k-1}\right\}=\{i \in N: \sigma(i)=\nu\} .
$$

Let $j \in N$ and $R_{j}^{\prime} \in \mathcal{R}$. We shall characterize the set $F\left(R_{j}^{\prime}, R_{-j}\right)$. Let $z^{\prime} \equiv\left(\sigma^{\prime}, m^{\prime}\right) \in F\left(R_{j}^{\prime}, R_{-j}\right)$.

Case (i) $j>k$ :

Subcase (i-i) $v_{k} \leq v_{j}^{\prime}$ : Then, $F(R)=F\left(R_{j}^{\prime}, R_{-j}\right)$. 
Subcase (i-ii) $v_{k-1} \leq v_{j}^{\prime}<v_{k}$ : Then, $\sigma^{\prime}(j)=\nu$ and $m_{j}^{\prime} \in\left[\frac{\ell}{n} v_{j}^{\prime}, \frac{\ell}{n} v_{k}\right]$.

Subcase (i-iii) $v_{k-2} \leq v_{j}^{\prime} \leq v_{k-1} \leq v_{k}$ : Then, $\sigma^{\prime}(j)=\nu$ and

$$
m_{k}^{\prime} \in\left[-\frac{n-\ell}{n} v_{k-1},-\frac{n-\ell}{n} v_{j}^{\prime}\right] \text { and } m_{k-1}^{\prime} \in\left[\frac{\ell}{n} v_{j}^{\prime}, \frac{\ell}{n} v_{k-1}\right] .
$$

Subcase (i-iv) $v_{j}^{\prime}<v_{k-2} \leq v_{k-1} \leq v_{k}$ : Then,

$$
m_{k}^{\prime} \in\left[-\frac{n-\ell}{n} v_{k-1},-\frac{n-\ell}{n} v_{k-2}\right] \text { and } m_{k-1}^{\prime} \in\left[\frac{\ell}{n} v_{k-2}, \frac{\ell}{n} v_{k-1} \cdot\right]
$$

Since

$$
\max \left(R_{j}, F(R)\right) I_{j}\left(\alpha,-\frac{n-\ell}{n} v_{k-1}\right) \text { and } \min \left(R_{j}, F(R)\right) I_{j}\left(\nu, \frac{\ell}{n} v_{k}\right),
$$

in either subcase, whenever $w \notin F(R), \min \left(R_{j}, F(R)\right) R_{j} w_{j}$.

Case (ii) $j=k$ :

Subcase (ii-i) $v_{j}<v_{j}^{\prime}$ : Then, $\sigma^{\prime}(j)=\alpha$ and $m_{j} \in\left[-\frac{n-\ell}{n} v_{k-1},-\frac{n-\ell}{n} v_{k+1}\right]$.

Subcase (ii-ii) $v_{k-1}<v_{j}^{\prime}<v_{j}$ : Then, $\sigma^{\prime}(j)=\alpha$ and $m_{j} \in\left[-\frac{n-\ell}{n} v_{j}^{\prime},-\frac{n-\ell}{n} v_{k-1}\right]$.

Subcase (ii-iii) $v_{j}^{\prime}=v_{k-1}$ : Then, either $w_{j}=\left(\alpha,-\frac{n-\ell}{n} v_{k-1}\right)$ or $w_{j}=\left(\nu, \frac{\ell}{n} v_{k-1}\right)$.

Subcase (ii-iv) $v_{j}^{\prime}<v_{k-1}$ : Then, $\sigma^{\prime}(j)=\nu$ and $m_{j} \in\left[\max \left\{v_{j}^{\prime}, v_{k-2}\right\}, \frac{\ell}{n} v_{k-1}\right]$.

As in Case (i), we have

$$
\max \left(R_{j}, F(R)\right) I_{j}\left(\alpha,-\frac{n-\ell}{n} v_{k-1}\right) \text { and } \min \left(R_{j}, F(R)\right) I_{j}\left(\nu, \frac{\ell}{n} v_{k}\right) .
$$

Thus, in either subcase, we have $\max \left(R_{j}, F(R)\right) R_{j} w_{j}$. Furthermore, whenever $w \notin F(R), \min \left(R_{j}, F(R)\right) R_{j} w_{j}$.

Case (iii) $j=k-1$ : Parallels to Case (ii).

Case (iv) $j<k-1$ : Parallels to Case (i).

Theorem 2 contrasts with many impossibility results in the literature. In particular, it is known that, when there are two agents, the class of singlevalued and strategy-proof solutions can be characterized by certain forms of constancy or dictatorship (Schummer, 2000, Theorem 1). Also, when there is only one real object, even on finitely restricted domains, there is no singlevalued subsolution of the Pareto solution satisfying strategy-proofness and equal compensation ${ }^{8}$ (Ohseto, 2000, Theorem 1). The no-envy solution is a subsolution of the Pareto solution that satisfies equal compensation and do not exhibit any constancy or dictatorship. Hence, our theorem implies that we can escape Schummer and Ohseto's impossibility theorems if we allow solutions to be multi-valued and extend strategy-proofness to extend strategy-proofness II.

\footnotetext{
${ }^{8}$ Every two agents who receive null objects should receive the same amount of money
} 


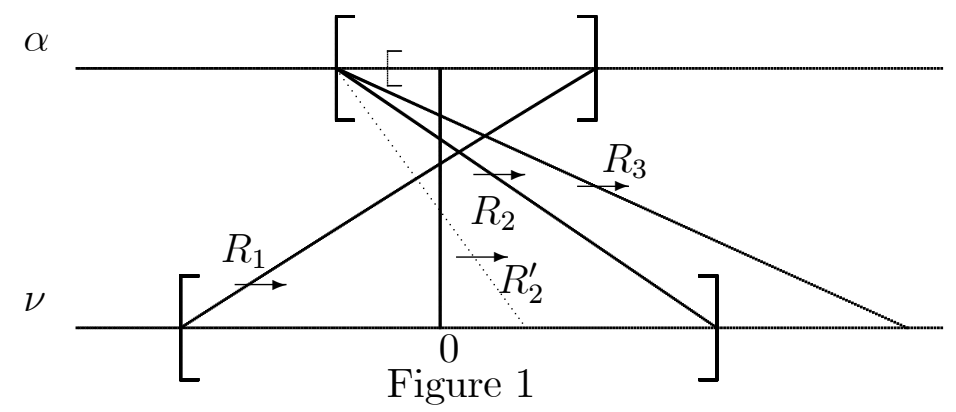

Though the no-envy solution satisfies extended strategy-proofness II, it is not immune to reduction manipulations. The next theorem characterizes the situations at which the no-envy solution is reduction-manipulable in terms of upper and lower thresholds.

Theorem 3. For each $R \in \mathcal{R}^{N}$, the no-envy solution is reduction-manipulable at $R$ if and only if $\bar{v}>\underline{v}$.

Proof. We prove this theorem only for the three agent case where $N=\{1,2,3\}$ and $v \equiv(-3,2,3)$. It can be easily generalized to any finite number of agents and any preference profile. Indeed, a general proof can be obtained in a similar way as Theorem 2 using Lemma 2, so we omit it.

By Lemma 2, any $(\sigma, m) \in F(R)$ is characterized by $\sigma(1)=\nu$ and

$$
m_{1} \in\left[-2, \frac{4}{3}\right] \text { and } m_{2} \in\left[-\frac{2}{3}, 1\right] \text {. }
$$

Figure 1 describes the set of envy-free allocations in this economy.

One important thing to observe in the figure is that the upper bound -in terms of utility - from agent 2 and 3's point of view is entirely determined by agent 1 , the agent who gets a null object. Indeed, this corresponds to the lower bound from agent 1's point of view. On the other hand, agent 2, the one with the second highest valuation for the real object determines the lower bound from agent 2 and 3's perspective. Consider agent $2^{9}$. Suppose he reports a valuation $v_{1}<v_{2}^{\prime}<v_{2}$. Since the valuation for the real object he reports is

\footnotetext{
${ }^{9}$ Agent 3 can indeed also reduction-manipulate the solution.
} 
lower, agent 2 cannot pay as much as before. The consequence is a shift in the lower bound of $m_{2}$ to $m_{2}^{\prime}$. That is,

$$
m_{2}^{\prime} \in\left[-\frac{2}{3}+\varepsilon, 1\right], \varepsilon>0 .
$$

Therefore, any agent who obtains a real object may reduction manipulate the no-envy solution. Also, observe that agents who obtain a null object can also reduction-manipulate the solution. By reporting $v_{1}^{\prime}<v_{1}$, agent 1 shift his own lower bound to the left so this is not profitable. But by reporting $v_{1}^{\prime}>v_{1}$, it does influence the lower bound of $m_{1}$ which changes to $m_{1}^{\prime}$,

$$
m_{1}^{\prime} \in\left[-2+\varepsilon, \frac{4}{3}\right], \varepsilon>0 .
$$

Indeed, the argument extends to any finite number of agents, any number of possible preference profiles and any number of real objects $\ell \leq n-1$. Notice that the reason why we need that $\bar{v}_{i}>\underline{v}_{i}$ is because the set of no-envy allocation is otherwise essentially singleton.

When there is only one real indivisible object, it is known that no subsolution of the no-envy solution satisfies extended strategy-proofness III (Tadenuma and Thomson, 1995, Theorem 1). The next theorem generalizes this impossibility result by allowing several real objects to exist and enlarging the no-envy solution to the Pareto and identical preferences lower bound solution:

Theorem 4. There is no subsolution of the Pareto and identical preferences lower bound solution satisfying extended strategy-proofness III.

Proof. Suppose, by contradiction, that there exists $\psi \subseteq P \underline{B}_{i p}$ satisfying extended strategy-proofness III. Let $R \in \mathcal{R}^{N}$ be such that

$$
v \equiv(\underbrace{1,1, \ldots, 1}_{\ell}, \underbrace{0,0, \ldots, 0}_{n-\ell}) .
$$

Let $z \equiv(\sigma, m) \in \psi(R)$. Since $z$ is Pareto efficient, $\sigma(1)=\alpha$.

Case 1. $m_{\mathbf{1}}<0$ : This is the the case that agent 1 prefers his optimal envy-free consumption bundle $(\alpha, 0)$ to $z_{i}$. Since $r\left(R_{i}\right)=\left(\alpha,-\frac{n-\ell}{n}\right)$,

$$
-\frac{n-\ell}{n} \leq m_{1}<0
$$

If agent 1 reports $v_{1}^{\prime}$ such that

$$
m_{1}<-\frac{n-\ell}{n} v_{1}^{\prime}<0
$$


then by $r\left(R_{1}^{\prime}\right)=\left(\alpha,-\frac{n-\ell}{n} v_{1}^{\prime}\right)$, for each $z^{\prime} \in \psi\left(R_{1}^{\prime}, R_{-1}\right), z_{1}^{\prime} P_{1} z_{1}$. Thus, agent 1 can manipulate $\psi$ at $z$.

Case 2. $\mathbf{0} \leq \boldsymbol{m}_{\mathbf{1}}$ : By $\psi \subseteq P \underline{B}_{i p}$, every $i \in\{1,2, \ldots, \ell\}$ receives $\left(\alpha, m_{i}\right)$ such that $m_{i} \geq-\frac{n-\ell}{n}$. This implies that there exists $i \in\{\ell+1, \ell+2, \ldots, n\}$ who prefers his optimal envy-free consumption bundle $\left(\nu, \frac{\ell}{n}\right)$ to $z_{i}$. Then, by the same logic as Case 1, this agent can manipulate $\psi$ at $z$.

Thus, for each $z \in \psi(R)$, there exists an agent who can manipulate $\psi$ at $z$. This contradicts extended strategy-proofness III.

Recall that the no-envy solution is a subsolution of the Pareto and identical preferences lower bound solution. Therefore, this theorem implies that none of the no-envy solution and its subsolutions satisfies extended strategyproofness III. However, if we replace extended strategy-proofness III to II, we instead obtain a possibility result, since the no-envy solution satisfies it.

We introduce a familiar fairness axiom: two agents whose preferences are the same should receive consumption bundles at which they are indifferent.

Equal treatment of equals: For each $R \in \mathcal{R}^{N}$, each $z \in \psi(R)$, and each $i, j \in N$, if $R_{i}=R_{j}$, then $z_{i} I_{i} z_{j}$ (and so $z_{i} I_{j} z_{j}$ ).

All subsolutions of $F, E, E^{\alpha}, E^{\nu}$ satisfy equal treatment of equals.

We also discuss a mild neutrality property introduced by Tadenuma and Thomson (1991): the names of objects should not matter: if an allocation is obtained from a chosen allocation by an indifferent permutation, then it should also be chosen. Given two allocations $z, z^{\prime} \in Z$ and a profile $R \in \mathcal{R}^{N}$, we write $z \simeq_{R} z^{\prime}$ if for each $i \in N, z_{i} I_{i} z_{i}^{\prime}$, and there is a bijection $\pi: N \rightarrow N$ such that for each $i \in N, z_{\pi(i)}=z_{i}^{\prime}$.

Neutrality: For each $R \in \mathcal{R}^{N}$, each $z \in \psi(R)$, and each $z^{\prime} \in Z$, if $z \simeq_{R} z^{\prime}$, then $z^{\prime} \in \psi(R)$.

Neutrality is justified in terms of informational restriction in aggregation. All solutions introduced in Section 2 are neutral. 2. 10

We obtain a negative result on extended strategy-proofness $I V$ when $n=$

Theorem 5. Assume that $n=2$. There exists no subsolution of the Pareto solution satisfying extended strategy-proofness IV, equal treatment of equals, and neutrality.

Proof. Suppose, by contradiction, that there exists $\psi \subseteq P$ satisfying extended strategy-proofness $I V$, equal treatment of equals, and neutrality.

\footnotetext{
${ }^{10}$ It is unknown whether this results can be extended to the case $n>2$.
} 
Let $R \in \mathcal{R}^{N}$ be such that $v \equiv(1,0)$. Since there are infinitely many envyfree allocations for $R$, if we show that for each $z \in F(R), \psi(R)=\{z\}$, this is a contradiction.

Let $z \in F(R)$. Let $R^{0} \in \mathcal{R}^{N}$ be such that $R_{1}^{0}=R_{2}^{0}$ and $z_{1} I_{1}^{0} z_{2}$ (and so $\left.z_{1} I_{2}^{0} z_{2}\right)$. By equal treatment of equals and neutrality, $\psi\left(R^{0}\right)=\left\{z^{\prime} \in Z\right.$ : For some $\left.\pi \in \Pi, z^{\prime}=\pi(z)\right\}$.

Step 1. $\psi\left(\boldsymbol{R}_{\mathbf{1}}, \boldsymbol{R}_{\mathbf{2}}^{\mathbf{0}}\right)=\{\boldsymbol{z}\}$ : Let $x \in \psi\left(R_{1}, R_{2}^{0}\right)$. If $x_{1} P_{1}^{0} z_{1}$, then by $x \in \psi\left(R_{1}, R_{2}^{0}\right) \backslash \psi\left(R^{0}\right)$, Part 2 of extended strategy-proofness $I V$ is not met. If $z_{1} P_{1}^{0} x_{1}$, then by $z_{1} P_{1} x_{1}$ and $x \in \psi\left(R_{1}, R_{2}^{0}\right) \backslash \psi\left(R^{0}\right)$, Part 1 of extended strategy-proofness $I V$ is not met. Hence, $x_{1} I_{1}^{0} z_{1}$. By Pareto efficiency, agent 1 receives $\alpha$ both at $z_{1}$ and $x_{1}$. Thus, $x_{1}=z_{1}$. Hence, $x_{2}=z_{2}$, so $x=z$.

Step 2. $\psi(\boldsymbol{R})=\{z\}$ : This step can be proved by an identical way to Step 1, so we omit it.

Remark 1. The impossibilities of Theorems 1, 4, and 5 hold even on more restricted preference domains. One can easily observe that it suffices that the domain of valuations is a real interval.

Remark 2. When there are several types of objects, the set of Pareto and egalitarian-equivalent allocations and the set of envy-free allocations do not always intersect (Thomson, 1990). One can easily observe from Lemma 3 that the same relation holds in our case.

\section{Conclusion}

1) The egalitarian-equivalent efficient and the no-envy solutions differ with respect to possible manipulations. On the one hand, results on the no-envy solution confirm that requiring single-valuedness may be too demanding. On the other hand, this intuition is not confirmed by the egalitarian-equivalent efficient solution.

2) The results show that we can escape some recent negative results (see e.g. Schummer, 1999; Svensson, 2000; Ohseto 2000 and 2003) in indivisible goods models. However, even the positive results on the no-envy solution have some limitations. We have been able to identify what kind of manipulations occur. Adding allocations to the selection by misrepresenting preferences cannot be profitable, but an agent can gain by eliminating some allocations. Thus, the positive results may be contrasted by the persistence of some form of manipulations. 
3) Extended strategy proofness III is very demanding. No subsolution of the Pareto and indentical preferences lower bound satisfy it.

4) Future work should seek to look at other possible definitions of extended strategy proofness and look at other fair solutions. Moreover, whether or not these results extend to other domains or other environments is not known yet. In particular, whether one obtains negative results in environments like exchange economies is an important question to address.

5) Extended strategy proofness I has been identified by Jackson (1992) as a necessary condition for implementation in undominated strategies using bounded mechanisms. Undominated strategies is also a very weak solution concept. We do not know yet what sufficiency requires. It would be of interest to derive a necessary and sufficient condition for solutions to be implementable in undominated strategies. The next step would then be to construct simple mechanisms to implement them.

\section{References}

Alkan, A., G. Demange, and D. Gale (1991) "Fair Allocation of Indivisible Goods and Criteria of Justice," Econometrica 59, 1023-1039.

Barbera S. (1977) "The Manipulation of Social Choice Mechanisms that Do Not Leave Too Much to Chance," Econometrica 45: 1573-1588.

Barbera S., Dutta B. and Sen A. (2001) "Strategy-Proof Social Choice Correspondences," Journal of Economic Theory 101: 374-394.

Bevia, C. (1996) "Identical Preferences Lower Bound Solution and Consistency in Economies with Indivisible Goods," Social Choice and Welfare 13: $113-126$.

Bevia, C. (1998) "Fair Allocation in a General Model with Indivisible Goods," Review of Economic Design 3: 195-213.

Ching S. and Zhou L. (2002) "Multi-Valued Strategy-Proof Social Choice Rules," Social Choice and Welfare 19: 569580.

Gibbard A. (1977) "Manipulation of Schemes that Mix Voting with Chance," Econometrica 45: 665-681.

Foley, D. (1967) "Resource Allocation and the Public Sector," Yale Economic Essays 7, 45-98. 
Jackson, M. (1992) "Implementation in Bounded Strategies: A Look at Bounded Mechanisms," Review of Economic Studies 59, 757-775.

Ohseto, S. (2001) "Strategy-proof and Efficient Allocation of an Indivisible Good on Finitely Restricted Domains," International Journal of Game Theory 29, 365-374.

Ohseto, S. (2004) "Implementing Egalitarian-equivalent Allocation of Indivisible Goods on Restricted Domains," Economic Theory 23, 659-670.

Schummer, J. (2000) "Eliciting Preferences to Assign Positions and Compensation," Games and Economic Behavior 30, 293-318.

Svensson, L.-G. (1983) "Large Indivisibilities: An Analysis with Respect to Price Equilibrium and Fairness," Econometrica 51, 939-954.

Svensson, L.-G. (2002) "Strategy-proof and Nonbossy Allocation of Indivisible Goods and Money," Economic Theory 20, 483-502.

Tadenuma, K., and W. Thomson (1991) "No-envy and Consistency in Economies with Indivisible Goods," Econometrica 59, 1755-1767.

Tadenuma, K., and W. Thomson (1993) "The Fair Allocation of an Indivisible Good When Monetary Compensations are Possible," Mathematical Social Sciences 25, 117-132.

Tadenuma, K., and W. Thomson (1995) "Games of Fair Division," Games and Economic Behavior 9, 191-204.

Tadenuma, K., and W. Thomson (1995b) "Refinements from the No-envy Solution in Economies with Indivisible Goods," Theory and Decision 39, 189-206.

Thomson, W. (2004) Theory of Fair Allocation, mimeo, University of Rochester. 